einer für die betroffenen Allergologen günstigeren Lösung übergehen.

\section{Rechtssicherheit für hessische Allergologen}

Jüngst hat die KV Hessen nun mitgeteilt, dass zukünftig die Möglichkeit besteht, Präparate zur SIT bei entsprechenden Nachweisen als Praxisbesonderheit anzuerkennen. Eine Pseudoziffer wurde hierfür zwar nicht geschaffen und ein Automatismus ist ohnehin aufgrund der gesetzlichen Vorgaben nicht möglich (der Prüfungsausschuss entscheidet hierüber, nicht die $\mathrm{KV}$ ), jedoch ist die grundsätzliche Akzeptanz der SIT als wirksame und wirtschaftliche Therapie schon als Erfolg zu bewerten. Im Rahmen einer „vorsorglichen Meldung" kann die Anzahl und Art der SIT durch den Arzt der KV gegenüber dokumentiert werden.

Voraussetzung für derartige Erfolge ist die kontinuierliche Bereitschaft engagierter Kollegen, persönliche Freizeit und letztlich auch erhebliche finanzielle Mittel aufzubringen. Hervorzuheben ist in diesem Zusammenhang Dr. Udo Rahmel, HNO-Arzt und Allergologe aus Hofheim, ohne dessen außergewöhnliches persönliches Engagement auch die Aktivitäten der Allergologenverbände nicht erfolgreich gewesen wären. Seine langjährige Arbeit für die gute Sache kann anderen Kollegen als Beispiel dafür dienen, dass sich Engagement und persönlicher Einsatz letztlich doch lohnen können: Der Ärzteverband Deutscher Allergologen dankt!

Prof. Dr. Ludger Klimek, Wiesbaden

\title{
MVZ liegen weiter im Trend
}

D er Trend zur Bildung Medizinischer Versorgungszentren (MVZ) in Deutschland hält an. Nach Angaben der Kassenärztlichen Bundesvereinigung ist die Anzahl der MVZ dieses Jahr erstmals auf über 1.000 angestiegen und lag zum Stichtag 30. Juni bei 1.088. Fast ein Viertel der Einrichtungen liegt in Bayern (233), es folgen Berlin (117) und Niedersachsen (103). In diesen Zentren arbeiten bereits 4.803 der insgesamt rund 140.000 ambulant tätigen Ärzte in Deutschland, im Durchschnitt vier pro MVZ. Zu 56\% sind die dort arbeitenden
Ärzte auch selbst Träger der Einrichtung. Was die Fachrichtung anbetrifft, so sind bundesweit Hausärzte, Internisten und Chirurgen am häufigsten vertreten.

Der erste Vorsitzende der KBV, Dr. Andreas Köhler, sieht die Entwicklung positiv: „Insbesondere junge Ärztinnen und Ärzte scheuen das Risiko einer selbstständigen Niederlassung. Sie können in einem MVZ als Angestellte in der ambulanten Versorgung arbeiten, Familie und Beruf wesentlich besser vereinbaren und später Partner oder Inhaber einer eigenen Praxis werden."
Ärztliche Ernährungsberatung erlaubt

Ein Hersteller von Diätprodukten versuchte, Ärzte als Ernährungsberater anzuheuern, um sein „Ernährungsprogramm zum Abnehmen“ zu vertreiben. „Erweitern Sie Ihr Leistungsspektrum“, forderte er in einem Internetauftritt die Mediziner auf. Das ärztliche Berufsrecht stehe gewerblicher Tätigkeit nicht entgegen, wenn diese außerhalb der Sprechstunden stattfinde. Kassen, Bankkonten sowie Sach- und Arbeitsmittel müssten von der Arztpraxis getrennt verwaltet werden.

Die Zentrale zur Bekämpfung unlauteren Wettbewerbs wandte sich gegen diese Werbung: Ernährungsberatung in Praxisräumen sei wettbewerbswidrig und verstoße gegen die Berufsordnung für Ärzte. Dem widersprach jetzt der Bundesgerichtshof: Das Unternehmen weise in der Werbung klar darauf hin, dass gewerbliche Ernährungsberatung und freiberufliche ärztliche Tätigkeit organisatorisch, wirtschaftlich und rechtlich voneinander getrennt durchzuführen seien. Wenn Mediziner diese Bedingungen erfüllten, verstoße die Ernährungsberatung weder gegen das Berufsrecht, noch sei sie wettbewerbswidrig.

Kommerzielle Gesichtspunkte müssten vom Heilauftrag des Arztes getrennt bleiben. Patienten müssten darauf vertrauen können, dass sich der Arzt bei der Behandlung nicht von gewerblichen Interessen leiten lasse, sondern von medizinischem Wissen. Gewerbliche Tätigkeit sei den Medizinern aber nicht grundsätzlich untersagt. Die Teilnahme an einem Diätprogramm würden Patienten nicht unbedingt als Anzeichen dafür werten, dass sich Mediziner zunehmend einem Gewerbe widmeten. gri

Bundesgerichtshof, I ZR 75/05

Sonderzahlungen: Freiwillig heißt freiwillig

Wird in einem Arbeitsvertrag darauf hingewiesen, dass durch eine freiwillige Sonderzahlung des Unternehmens kein Rechtsanspruch der Arbeitnehmer darauf entsteht, dass diese Leistung auch in Zukunft gezahlt wird, ist die Klausel mit diesem (Freiwilligkeits-)Vorbehalt voll wirksam. Sie benachteiligt die Mitarbeiter nicht unangemessen. gri 